Kinder- und Jugendkriminalität 
René Bendit/Wolfgang Erler/Sima Nieborg/ Heiner Schäfer (Hrsg.)

\section{Kinder- und Jugendkriminalität}

Strategien der Prävention und Intervention in Deutschland und den Niederlanden

Springer Fachmedien Wiesbaden GmbH 2000 
Die Vorbereitung dieser Publikation wurde durch Fördermittel des Bundesministeriums für Familie, Senioren, Frauen und Jugend ermöglicht.

ISBN 978-3-8100-2382-7 ISBN 978-3-663-10471-1 (eBook)

DOI 10.1007/978-3-663-10471-1

Gedruckt auf säurefreiem und alterungsbeständigem Papier.

Die Deutsche Bibliothek - CIP-Einheitsaufnahme

Kinder- und Jugendkriminalität : Strategien der Prävention und Intervention in Deutschland und den Niederlanden / Hrsg.: René Bendit ; Wolfgang Erler ; Sima Nieborg ; Heiner Schäfer. - Opladen : Leske + Budrich, 2000

NE: Bendit, René [Hrsg.];

(C) 2000 Springer Fachmedien Wiesbaden

Ursprünglich erschienen bei Leske + Budrich, Opladen 2000.

Das Werk einschließlich aller seiner Teile ist urheberrechtlich geschützt. Jede Verwertung außerhalb der engen Grenzen des Urheberrechtsgesetzes ist ohne Zustimmung des Verlages unzulässig und strafbar. Das gilt insbesondere für Vervielfältigungen, Übersetzungen, Mikroverfilmungen und die Einspeicherung und Verarbeitung in elektronischen Systemen. 


\section{Inhalt}

Editorial

Ingo Richter/Jan Willem Duyvendak

Vorwort.

Bernd Holthusen/Heiner Schäfer

Einführung: Prävention von Kinder- und Jugenddelinquenz

in Deutschland und in den Niederlanden

Erster Teil: Kinder- und Jugenddelinquenz in den Niederlanden und Deutschland:

Möglichkeiten und Grenzen präventiver Ansätze

1 Präventionsstrategien aus kommunalpolitischer Sicht:

Zwei Beispiele

1.1 Ton Peters

Delinquenz und Prävention in Rotterdam

1.2 Friedrich Graffe

Delinquenz und Prävention in München

2 Kinder- und Jugenddelinquenz: Präventionsstrategien vor dem Hintergrund neuer sozialer Herausforderungen

2.1 Christian von Wolffersdorff

Jugendkriminalität in Deutschland - Über den Umgang mit schwierigen

Jugendlichen und das neue Bedürfnis nach ,law and order“

2.2 Peter van der Laan

Jugendkriminalität in den Niederlanden: Neue Konzepte,

neue Herausforderungen, neue Einsichten

Zweiter Teil: Berichte aus der Praxis Präventive Projekte zur Kinder- und Jugenddelinquenz

3 Aufwachsen unter schwierigen Lebensbedingungen: Soziale Deprivation, ethnische Konflikte und Kriminalprävention

3.1 Rudolf Pettinger

Zur öffentlichen Diskussion von Kinder- und Jugenddelinquenz in

Deutschland - eine Einführung 
3.2 Erik Snel/Henk Ferwerda

Jugendkriminalität in benachteiligten Wohnvierteln

3.3 Anton Schrama

AGADIR - ein Interventionsprojekt für gefährdete Jugendliche

3.4 Siep Buist

Gebietsbezogene Gerichtsbarkeit (JIB) in Arnhem

3.5 Alp Otman

Eltern- und Familienarbeit in Migranten-,,communities“ im Rahmen der Prävention von Jugenddelinquenz

3.6 Gerd-Rüdiger May

Sozialpädagogische Familienhilfe und ihr Einfluß auf

Kinder- und Jugenddelinquenz

3.7 Martin Rehse

Integrative Familienhilfe - Familienhilfe als Möglichkeit

zur Kriminalprävention

3.8 Jean-Marc Felber

Die präventiven Maßnahmen der „Centres Socio-Culturels“ in

Frankreich - am Beispiel eines Stadtteils in Straßburg...

3.9 Tom Cockburn

Wie Jugendliche aus einem „Problemquartier“ in Manchester

ihre Situation einschätzen

3.10 Rudolf Pettinger

Schlußfolgerungen

4 Jugendkriminalität: Reaktionen auf Straftaten Jugendlicher und Möglichkeiten der Rückfallvermeidung

4.1 Bernd Holthusen/Stan Meuwese

Reaktionen auf Straftaten: Wie kann Rückfall vermieden werden eine Einführung

4.2 Claudio Morelli/Annemieke Wolthuis

Die Unterschiede zwischen dem niederländischen und dem deutschen Jugendstrafrecht

4.3 Gert Delattre

Der Täter-Opfer-Ausgleich - ein Modell zur

Wiedergutmachung im Strafverfahren 
4.4 Alma van Hees

Die HALT-Büros: Schnelle Reaktion auf Jugendkriminalität.

4.5 Ron Gerrits

Die T-Teams - Intensive ambulante Betreuung delinquenter

Kinder und Jugendlicher

4.6 Reinhard Koch/Thomas Möbius

Voneinander lernen: Der Transfer der niederländischen

INSTAP-Methode in das deutsche Jugendhilfesystem

4.7 BerndHolthusen/Stan Meuwese

Schlußfolgerungen

5 Jugendhilfeplanung als Teil einer Präventionsstrategie

5.1 Eric van Santen/Alice van Unen

Herausforderungen einer präventiven Jugendpolitik - eine Einführung ...... 185

5.2 Alice van Unen

Jugendhilfeplanung im niederländischen und deutschen

Jugendhilferecht - ein Vergleich

5.3 Eric van Santen

Anspruch und Wirklichkeit - Jugendhilfeplanung in der

Bundesrepublik Deutschland

5.4 Frits Vlek

Die Überwachung von Kinder- und Jugenddelinquenz und gefährdeten

Jugendlichen: Verbesserter Informationsstand als Voraussetzung

effektiver Prävention. 206

5.5 Maria Blom

Monitoring in Groningen und Bremen auf lokaler

und internationaler Ebene

5.6 Doris Pleiger

Kriminalpräventive Räte. Das Beispiel Rostock

5.7 Cor van 't Hoff

Informationssysteme: Lokale und nationale Trends in den Niederlanden....233

5.8 Wiebke Steffen/Erich Elsner

Kinder- und Jugenddelinquenz in München -

Befunde, Reaktionen und Maßnahmen 
5.9 Maria Pannebakker

Nachbarschaftsnetzwerke als Mittel der Früherkennung

delinquenten Verhaltens.

5.10 Eric van Santen/Alice van Unen

Schlußfolgerungen .256

6 Empowerment statt Ausgrenzung:

Ein neuer Ansatz in der Kriminalitätsprävention

6.1 Sabine Pankofer/Jo Hermanns

Empowerment statt Ausgrenzung schwieriger Jugendlicher -

eine Einführung...

6.2 Norbert Herriger

Empowerment in der pädagogischen Arbeit mit „Risiko-Jugendlichen“ .....263

6.3 Jo Hermanns/Heleen van der Stege

Strategien zur Integration straffälliger Jugendlicher

6.4 Rainer Gerhard/Etta Fennekohl

Gemeinschaftsgrundschule Roncallistraße - Stadtteilkonferenz.

Ein Stadtteil hilft seinen Kindern

6.5 Yvonne Duivenvoorden

Das SPRINT-Projekt: Die Stärkung von positivem Verhalten

und der Ausbau von Kompetenzen Jugendlicher

6.6 Martina Liebe

Das Aktionsprogramm „Präventive Jugendarbeit“"

des Bayerischen Jugendrings: Konzept und Erfahrungen

6.7 Martina Blug

Aufsuchende Jugendarbeit auf dem Land:

Empowerment und Prävention durch Vermittlung

6.8 Hyacinth Dashorn

„Neue Perspektiven" in Amsterdam.

6.9 Peter Heemann

Jugendhilfe meets Drogenhilfe.

Oder: Das „Frankfurter Crack-Street-Projekt““.

6.10 Rob Rutten

Das Projekt „Die Chance“ (De Kans) in Den Haag.

6.11 Sabine Pankofer/Jo Hermanns

Schlußfolgerungen 
7 Berichte aus weiteren europäischen Ländern

7.1 René Bendit/Sima Nieborg

Probleme der Kinder- und Jugendkriminalität und Anforderungen an die

Jugendpolitik in anderen europäischen Ländern

7.2 Heide Tebbich

Gefährdete Jugendliche - Kinder- und Jugenddelinquenz

und Prävention in Österreich

7.3 Galina Kouprianova

Kinder- und Jugenddelinquenz und Jugendpolitik in Russland

7.4 Jackie Russell

Bürgerschaftliche Verantwortung, Empowerment.

Kriminalitätsprävention in Birmingham.

7.5 Ferran Casas/Teresa Crespo/Anna Tarrés/Sergi Pacual

Riskantes Freizeitverhalten von Jugendlichen

\section{Dritter Teil: Kinder- und Jugenddelinquenz im jugendpolitischen Kontext}

8 Jugendpolitische Perspektiven

8.1 Reinhard Wabnitz

Kinder und Jugendkriminalität. Aktuelle Herausforderungen

und Handlungsstrategien aus deutscher Sicht

8.2 Frans de Jong

Kinder und Jugendkriminalität. Aktuelle Herausforderungen

und Handlungsstrategien aus niederländischer Sicht

9 Ausblick

Heiner Schäfer/Bernd Holthusen

Kriminalitätsprävention: Lokale Aufgabe und

grenzüberschreitender Austausch.

Verzeichnis der Autorinnen und Autoren 


\section{Editorial}

\section{René Bendit, Wolfgang Erler, Sima Nieborg, Heiner Schäfer Deutsches Jugendinstitut, München/Verwey-Jonker-Institut, Utrecht}

In vielen europäischen Ländern, einschließlich Deutschland und den Niederlanden, hat eine neue Diskussion um die Kinder- und Jugendkriminalität eingesetzt. In ihr geht es nicht nur um Jugendpolitik und um gesetzliche Maßnahmen, die nötig sind, um mit der steigenden Anzahl von Kindern und Jugendlichen umzugehen, die in den Statistiken als „kriminell“ auftauchen. Vielmehr finden diese Diskussionen vor dem Hintergrund zunehmender sozialer Probleme und Unsicherheiten (Arbeitslosigkeit, Konflikte ethnischer Gruppen, Armut von Kindern und soziale Ausgrenzung) statt. Zentral sind in diesen Diskussionen Fragen um die Früherkennung, die Prävention, die Intervention, aber auch um den verstärkten Einsatz repressiver Maßnahmen in der Auseinandersetzung mit der Jugendkriminalität. Darüber hinaus begründet sich das gewachsene öffentliche Interesse in Politik und Wissenschaft für die Themen „Früherkennung“, „Prävention“ und „Neue Methoden der Intervention" sowohl aus den (unbefriedigenden) Ergebnissen der traditionellen Strategien und Methoden (einschließlich der polizeilichen und juristischen Verfahren) im Umgang mit Jugendkriminalität als auch aus der zunehmenden Kritik an den unzureichenden Ergebnissen moderner Strategien der Diversion sowie der außergerichtlichen und sozialarbeiterischen Präventionsbemühungen.

Unter diesen Umständen überrascht es nicht, daß es mehr Fragen als Antworten gibt, daß sich neue Herausforderungen an Politik und Wissenschaft stellen. Früherkennung und Prävention können z.B. nicht lediglich mit einer frühzeitigen Diagnose individueller Entwicklungen und rechtzeitigen Hilfen gleichgesetzt werden, sondern erfordern Konzepte, die sich mit (sozialen) Umwelteinflüssen und der Einbettung in soziale Netzwerke, Nachbarschaften, Quartiere, mit lokaler Jugendhilfeplanung, mit „Empowerment“ und mit innovativer Intervention befassen.

Der vorliegende Bericht dokumentiert die Beiträge, Diskussionen und Ergebnisse der zweiten deutsch-holländischen Konferenz „Youth at Risk“ „gefährdete Jugend“, die im Juni 1998 in Köln stattgefunden hat und deren Thema „Jugendkriminalität: Möglichkeiten der frühzeitigen Erkennung, Prävention und Intervention" war. An der Konferenz nahmen Fachleute aus Jugendhilfe und -politik, aus Wissenschaft und Praxis teil.

Hauptziel der Konferenz war es, das Wissen um den Zusammenhang zwischen den Straftaten von Kindern und Jugendlichen und deren immer schwierigeren alltäglichen Lebensbedingungen zusammenzutragen und zu diskutieren. Die Gefahren und Risiken des Aufwachsens von Kindern und Jugendlichen und das abweichende oder kriminelle Verhalten wurden miteinander in 
Verbindung gebracht und interpretiert: im Kontext der Lebensbedingungen von Kindern und Jugendlichen, ihrer Partizipationsmöglichkeiten in Familie und Schule, der gemeinsam verbrachten Zeit mit Altersgenossen und der Projekte der Jugendarbeit. Die Konferenzteilnehmer haben versucht, das Phänomen „Jugendkriminalität“ zu entdramatisieren und der Verzerrung der Diskussionen in der Öffentlichkeit entgegenzutreten. Die professionelle Diskussion konzentrierte sich daher auf die Erfahrungen aus Projekten in beiden Ländern, die mit der Früherkennung von sowie der Prävention/Intervention bei Jugendkriminalität befasst sind. Solche innovativen Modelle wurden vor dem Hintergrund ihrer konkreten lokalen Bedingungen, innerhalb derer sie entwickelt und/oder umgesetzt wurden, diskutiert. Um aber auch einen Überblick über Jugendkriminalität sowie Präventions- und Interventionsstrategien außerhalb der Niederlande und Deutschlands zu gewinnen, wurden Erfahrungen aus anderen europäischen Ländern einbezogen.

Die Konferenz trägt dazu bei, den 1996 auf der ersten Konferenz „Youth at Risk - gefährdete Jugend" in Noordwijkerhout begonnenen Informationsund Erfahrungsaustausch zwischen Expertinnen und Experten zu vertiefen, zu erweitern und in die praktische Arbeit umzusetzen. Die - wenn auch nur vorläufige - Auswertung der beiden Konferenzen zeigt, dass inzwischen eine große Zahl direkter Kontakte - sowohl formell als auch informell - , aber auch ein Austausch über virtuelle Medien stattgefunden haben. Dieser Austausch hat mit dazu beigetragen,

- einen regelmäßigen Informations- und Wissensaustausch zu unterstützen;

- die Kooperation zwischen deutschen, holländischen und anderen Experten auf den Gebieten „Jugendpolitik“, „Jugendhilfe“ und verwandten Forschungsgebieten zu verbessern; und

- Netzwerke zwischen zwei oder mehreren Ländern zu unterstützen, und das Wissen über die Arbeitshypothesen der „anderen“, deren organisatorische Strukturen, deren bisherige praktische Erfolge und deren Präventions- und Interventionsstrategien zu erleichtern.

Wie schon in der ersten deutsch-holländischen Konferenz (siehe Hermanns/Leu 1998), werden sich auch aus dieser weitere gemeinsame Projekte ergeben. Der nun vorgelegte Bericht soll dies über den Kreis der Teilnehmerinnen und Teilnehmer hinaus anregen. Schließlich geben die Themen und Diskussionen der Konferenz Gelegenheit und Anregungen zu europaweiten Auseinandersetzungen mit Themen und Fragen, die sich mit Jugendkriminalität und ,guter Praxis“ auf den Gebieten der Früherkennung, der Prävention und der Intervention befassen.

Die Herausgeber freuen sich, diesen Bericht nun der Allgemeinheit in Deutschland und den Niederlanden (und natürlich auch interessierten Leserinnen und Lesern aus anderen europäischen Ländern) vorstellen zu können. Wir möchten uns beim Bundesministerium für Familien, Senioren, Frauen und Jugend (BMFSFJ) und beim Holländischen Ministerium für Gesundheit, 
Soziales und Sport (VHS) für ihre großzügige Unterstützung bedanken nicht nur bei der Organisation der Konferenz, sondern auch bei der Publikation dieser Konferenzbeiträge. Auch möchten wir den Teilnehmerinnen und Teilnehmern danken - den Referentinnen und Referenten sowie denen, die die Arbeitsgruppenergebnisse präsentiert haben. Sie alle haben die Konferenz mit ihren theoretischen und praktischen Beiträgen bereichert.

Und schließlich möchten wir den deutschen und holländischen Moderatorinnen und Moderatoren der Arbeitsgruppen unseren besonderen Dank aussprechen: Dr. Rudolf Pettinger und Dr. Eric Snel (Arbeitsgruppe I), Bernd Holthusen und Stan Meuwese (Arbeitsgruppe II), Eric van Santen und Alice van Unen (Arbeitsgruppe III), Prof. Dr. Sabine Pankofer und Prof. Dr. Jo Herrmanns (Arbeitsgruppe IV). Ihr umfangreiches Wissen und ihre intensive kommunikative und organisatorische Arbeit während und nach der Konferenz waren eine unverzichtbare Voraussetzung in der Erstellung dieses Berichts. Gemeinsam mit den Koordinatoren der Konferenz, Dr. René Bendit, Wolfgang Erler, Siema Nieborg und Heiner Schäfer übernehmen sie die Verantwortung für die Wiedergabe der Ideen und Erfahrungen, die in den verschiedenen Kapiteln dieses Buches dokumentiert sind.

\section{Literatur}

Hermanns, J./Leu, H.R. (eds.) (1998): Familiy Risks and Familiy Support. Delft 


\section{Vorwort}

\section{Ingo Richter/Jan Willem Duyvendak Deutsches Jugendinstitut, München/Verwey-Jonker-Institut, Utrecht}

In diesem Band werden die (zum Teil gekürzten und überarbeiteten) Beiträge einer Fachtagung des Deutschen Jugendinstituts und des Verwey-JonkerInstituts aus Utrecht in den Niederlanden im Juni 1998 in Köln einer breiteren Fachöffentlichkeit zugänglich gemacht. Mit dieser Tagung und der vorliegenden Publikation wird die produktive Kooperation fortgesetzt, die sich bereits im September 1996 bei der Deutsch-Niederländischen Konferenz „Youth at Risk - gefährdete Jugend" in Noordwijkerhout bewährt hat. Standen damals die Risikofaktoren im Mittelpunkt, mit denen Jugendliche konfrontiert sind, so ist es hier eine spezielle Gruppe von Jugendlichen: delinquente Jugendliche.

Straftaten und insbesondere gewalttätiges Handeln von Kindern und Jugendlichen machen Schlagzeilen, sie sind in der letzten Zeit geradezu zu einem „Dauerbrenner" in den Medien geworden. Zur Illustration sei auf einige Überschriften von Zeitungsartikeln aus der deutschen Presse hingewiesen:

Jugendliche - oft rücksichtslos und brutal. Die Polizei registrierte im vergangenen Jahr fast 4000 Gewaltdelikte (der Bericht bezog sich auf München) - bei Minderjährigen sinkt die Hemmschwelle.

Die Gewalt nimmt weiter zu. Das geht aus dem Jahresbericht des Jugendamtes für das Jahr 1997 hervor.

Der Krieg der Jugendlichen.

Da bleibt keine Nase heil. Brutalität unter Jugendlichen ist nicht länger eine Domäne von Jungen - immer mehr Mädchen prügeln und foltern. (...)

Es sind unsere Kinder. Gewalt unter Jugendlichen - ein Abbild der Gesellschaft.

Nehmt die Vandalen in die Pflicht; Strategien gegen die wachsende Jugendkriminalität gesucht: Was wir von unseren Nachbarn lernen können.

„Schließt sie weg, die kriminellen Kids“. Wer macht Jugendkriminalität zum Thema?

Prügeln und geprügelt werden; Wie eine Großstadt bislang noch vergeblich nach dem besten Rezept sucht, um die wachsende Jugendkriminalität einzudämmen.

Angesichts der in diesen Schlagzeilen sichtbar werdenden Dramatisierung von Jugendkriminalität verwundert es nicht, daß Ängste und Verunsicherungen in der Bevölkerung wachsen. Politik, Öffentlichkeit, Sicherheitskräfte, Schulen und Jugendhilfeeinrichtungen äußern sich besorgt über den deutlichen statistischen Anstieg von Jugendkriminalität und insbesondere von Ge- 
waltdelikten. Denn auch wenn man diese Daten teilweise auf ein verändertes Anzeigeverhalten zurückführt, sie als Effekt einer modifizierten statistischen Erfassung oder einer erhöhten Sensibilität gegenüber Aggressionen und „abweichendem" Verhalten betrachtet - was früher als jugendliche Rüpelei galt, wird heute als Gewaltakt interpretiert -, so läßt sich ein akuter Handlungsbedarf angesichts des überproportionalen Anstiegs von Kinder- und Jugenddelinquenz in den letzten Jahren doch nicht leugnen. Insbesondere die sich in Gewaltakten äußernde Fremdenfeindlichkeit erregt öffentliches Aufsehen und gibt Anlaß zur Besorgnis.

Ganz ähnliche Entwicklungen finden in den Niederlanden statt: Eine Zunahme von Jugendkriminalität und -gewalt laut Statistik und ein lautstarkes Medienecho darauf, das sich in öffentliche Diskussionen und politische Reaktionen umsetzt.

Welche Maßnahmen geeignet sind, delinquentem Verhalten vorzubeugen, es abzubauen und zu verhindern, läßt sich nur vor dem Hintergrund systematischer und detaillierter Kenntnisse über seine Entstehung und Entwicklung beantworten. Im Deutschen Jugendinstitut sind in diesem Zusammenhang Forschungsprojekte entstanden, die sich mit Kinderdelinquenz, mit den affektiven Grundlagen für Fremdenfeindlichkeit und Gewalt sowie mit dem Umgang von Mädchen mit Gewalt in jugendgruppenspezifischen $\mathrm{Zu}-$ sammenhängen befassen. Ziel dieser Projekte ist es, die Rahmenbedingungen und Erscheinungsformen delinquenten Verhaltens bei Jugendlichen zu analysieren sowie die Leistungen der Jugendhilfe zur Eindämmung delinquenter Entwicklungen zu erfassen. Die daraus resultierenden Erkenntnisse sollen dazu beitragen, der Jugendpolitik Möglichkeiten und Wege aufzuzeigen, wie Jugenddelinquenz präventiv entgegengewirkt werden kann. Nun gibt es in Deutschland bereits in einer Vielzahl von Projekten - z.B. im Rahmen der Jugend- und Familienhilfe, der Schule, der berufsbezogenen Sozialarbeit und der Freizeitpädagogik - Ansätze zur Prävention von Kinder- und Jugendkriminalität. Sie sind jedoch meist voneinander isoliert, nehmen einander kaum zur Kenntnis und widmen sich unterschiedlichen Zielgruppen. Wo Lücken bestehen, ob die angestrebten Wirkungen und Effekte erreicht werden, ist ebenfalls ungeklärt. Zur Beseitigung derartiger Defizite will die „Arbeitsstelle Kinder- und Jugendkriminalität" im Deutschen Jugendinstitut beitragen. Ihr Ziel ist es, einen Überblick über die vorhandenen Präventionsmaßnahmen zu geben, Informationen über erprobte und bewährte Konzepte, Strategien und Arbeitsformen $\mathrm{zu}$ verbreiten, die Wirksamkeit in regionalen und lokalen Kontexten für unterschiedliche Zielgruppen zu überprüfen, Evaluationsmaßstäbe zu erarbeiten, die Vernetzung der einzelnen Träger und Institutionen zu fördern und den Austausch von Praxiserfahrungen zwischen den Projekten anzuregen. 
Mit der Darstellung unterschiedlicher europäischer Ansätze verknüpfen wir die Hoffnung und Erwartung, daß sich die Zusammenarbeit über nationale Grenzen und unterschiedliche Jugendhilfesysteme hinaus intensiviert und daß die unterschiedlichen Ansätze aus Wissenschaft, Politik- und Praxis gegenseitige Lernprozesse befördern, die uns helfen, junge Menschen bei der Bewältigung ihrer spezifischen Entwicklungsaufgaben besser zu unterstützen und so der Entwicklung von Kriminalität und Gewalt entgegenzuwirken. 